\title{
Cancro Anal em Doentes com Infecção por VIH - Experiência do Hospital de Curry Cabral
}

\author{
Diana Póvoas' ${ }^{1}$ Cândida Fernandes², Vasco Macias33, Bárbara Flor de Lima', Ana Rodrigues², Fernando Maltez , Jorge Cardoso 5 \\ 'Interno de Doenças Infecciosas/Resident, Infectious Diseases, Serviço de Dermatologia e Venereologia, Hospital de Curry Cabral, \\ Centro Hospitalar de Lisboa Central, Lisboa, Portugal \\ ${ }^{2}$ Assistente Hospitalar Graduado de Dermatovenereologia/Consultant, Dermatology, Serviço de Dermatologia e Venereologia, \\ Hospital de Curry Cabral, Centro Hospitalar de Lisboa Central, Lisboa, Portugal \\ ${ }^{3}$ Assistente Hospitalar de Dermatovenereologia/Consultant, Dermatology, Serviço de Dermatologia e Venereologia, Hospital de \\ Curry Cabral, Centro Hospitalar de Lisboa Central, Lisboa, Portugal \\ ${ }^{4}$ Chefe de Serviço, Director do Serviço de Doenças Infecciosas/Consultant Chief, Head of the Department of Infectious Diseases, \\ Hospital de Curry Cabral, Centro Hospitalar de Lisboa Central, Lisboa, Portugal \\ ${ }^{5}$ Chefe de Serviço, Director do Serviço de Dermatologia e Venereologia/Consultant Chief, Head of the Department of Dermatology, \\ Hospital de Curry Cabral, Centro Hospitalar de Lisboa Central, Lisboa, Portugal
}

RESUMO - Introdução: Os homens que têm sexo com homens, particularmente os infectados pelo vírus da imunodeficiência humana $(\mathrm{VIH})$ têm risco aumentado de cancro anal. Ao contrário da maior parte das doenças oportunistas e/ou definidoras de SIDA, os dados são contraditórios no que diz respeito ao efeito da terapêutica antirretrovírica, não sendo claro o impacto da supressão virológica e reconstituição imunitária na evolução da infecção por vírus do papiloma humano (HPV) e prevenção das neoplasias associadas. Actualmente é já recomendada a vacinação de doentes com infecção por VIH contra o HPV; os programas de rastreio do cancro anal, recomendados por algumas organizações, carecem ainda de validação definitiva da sua eficácia. Material e Métodos: Os autores fizeram um estudo retrospectivo dos casos de cancro anal diagnosticados entre 2000-2015 em doentes com infecção por VIH em seguimento no serviço de Infecciologia do Hospital de Curry Cabral, em Lisboa. São avaliadas as características clínicas e epidemiológicas dos doentes com cancro do canal anal ao longo de um período de quinze anos. Resultados: $O$ cancro anal foi diagnosticado em dez doentes, maioritariamente homens $\mathrm{HSH}$, com infecção por VIH com uma duração média de 15, 1 anos, com contagem média de linfócitos TCD4+ de 441 células/uL. O diagnóstico foi mais frequentemente realizado no estádio III e o tratamento mais frequente foi cirúrgico, ocasionalmente conjugado com radioterapia. Faleceram 4 doentes. Conclusões: São necessários programas de rastreio consensuais do cancro anal, a par da necessidade de reforço da vacinação contra o HPV nos homens, nomeadamente nos homens que têm sexo com homens, com ou sem infecção por VIH.

PALAVRAS-CHAVE - Homossexualidade Masculina; Infecções por VIH; Infecções por Papilomavirus; Neoplasias do Ânus.

\section{Anal Cancer in HIV Patients - Experience at Hospital de Curry Cabral}

ABSTRACT - Introduction: Men who have sex with men, particularly those infected by human immunodeficiency virus (HIV), have an increased risk of anal cancer. Unlike most opportunistic or AIDS-defining events, data have been contradictory regarding impact of antiretroviral therapy, viral suppression and immunologic reconstitution on HPV infection prevention and related cancers. Vaccination against human papillomavirus (HPV) is presently recommended in all HIV-infected patients, whereas there is still an on-going debate about the need for anal cancer screening programmes. Material and methods: The authors performed a retrospective study of anal cancer cases diagnosed between 2000-2015 in HIV patients on follow up at the Infectious Diseases Unit at Hospital de Curry Cabral, in Lisbon. We present the clinical and epidemiological characteristics of anal cancer cases throughout a fifteen-year period. Results: Anal cancer was diagnosed in ten patients, most of them MSM, HIV-infected for an average time of 15.1 years, with an average TCD4 + cell count of de 441 cells/uL. Anal cancer diagnosis was more frequently performed at stage III and treatment most

Correspondência: Diana Póvoas

Serviço de Doenças Infecciosas - Hospital de Curry Cabral

Rua da Beneficência, 8 - 1069-166 Lisboa, Portugal - Tel: 217924280 - Fax: 217924281

E-mail: d.povoas@gmail.com
Recebido/Received

9 Novembro/9 November 2015

Aceite/Accepted

18 Janeiro/18 January 2016 
frequently involved surgery, occasionally with radiotherapy. Four patients died. Conclusion: There is a need for consensual anal cancer screening programs, along with a need to reinforce HPV vaccination in men, particularly men who have sex with men, regardless of HIV infection.

KEY-WORDS - Anus Neoplasms; HIV Infections; Homosexuality, Male; Papillomavirus Infections.

\section{INTRODUÇÃO}

As neoplasias relacionadas com a infecção pelo vírus do papiloma humano (HPV) assumem particular importância nos doentes com infecção pelo vírus da imunodeficiência humana $(\mathrm{VIH})$, tendo-se vindo a registar um aumento na frequência de cancro anal nesta população, mais frequentemente em homens que têm sexo com homens (HSH). ${ }^{1,2}$

\section{MATERIAL E MÉTODOS}

Foi feito um estudo retrospectivo dos casos de cancro anal diagnosticados entre 2000-2015 em doentes com infecção por VIH em seguimento no serviço de Infecciologia do Hospital de Curry Cabral. Os doentes foram identificados a partir dos registos dos diagnósticos de neoplasias não definidoras de SIDA dos doentes com infecção por VIH em seguimento. Os processos clínicos foram revistos, tendo sido recolhidos dados relativos a características demográficas, tempo de diagnóstico de infecção por $\mathrm{VIH}$, estádio imunológico e virológico na altura dos diagnósticos de infecção por $\mathrm{VIH} \mathrm{e}$ cancro do canal anal, terapêutica antirretrovírica, história passada de processos oportunistas, características clínicas, procedimentos de diagnóstico e terapêutica, estadiamento da doença neoplásica, evolução clínica e mortalidade.

O diagnóstico de cancro anal foi feito por histologia; a detecção de HPV foi realizada por amplificação por reacção em cadeia da polimerase (PCR) em tecidos embebidos em parafina utilizando o dispositivo CLART $^{\circledR}$ Papilomavírus
Humano, que detecta 35 tipos de $\operatorname{HPV}(6,11,16,18,26,31$, $33,35,39,40,42,43,44,45,51,52,53,54,56,58,59$, $61,62,66,68,70,71,72,73,81,82,83,84,85$ e 89). 0 estadiamento foi feito de acordo com o sistema internacional TNM. A mortalidade global foi definida como o total de óbitos ocorridos durante o período em análise.

\section{RESULTADOS}

Num universo de 2497 doentes com infecção por VIH em seguimento no serviço de Infecciologia do Hospital de Curry Cabral, entre 2000-2015 foram diagnosticados 10 casos de neoplasia do canal anal (Tabela 1), correspondendo na totalidade a homens, idade média de 49,5 anos (32-81 anos), a maioria $\mathrm{HSH}(\mathrm{n}=8)$. No momento do diagnóstico de cancro anal, o tempo de infecção por VIH era em média de 15,1 anos (mediana 16, 5-20 anos), com contagem de linfócitos TCD4+ média de 441 células/uL (mediana 403, 6-930 células/uL). Dois doentes tinham carga viral (CV) detectável, um em interrupção de terapêutica antirretrovírica (TARV). Os restantes nove casos encontravam-se sob TARV, todos com CV indetectável, excepto um doente, com CV inferior a 1000 cópias $/ \mathrm{mL}$. Três doentes tinham história de doença oportunista, tuberculose $(n=2)$ e sarcoma de Kaposi cutâneo $(n=1)$. Nenhum tinha recebido vacinação contra o HPV.

O quadro clínico mais frequentemente detectado incluiu queixas locais, como a presença de placas ou lesões vegetantes $(n=6)$, rectorragia $(n=4)$, proctalgia $(n=3)$, tenesmo

\section{Tabela 1 - Características demográficas, clínicas e estadiamento de acordo com o sistema TNM dos casos de cancro do canal anal.}

\begin{tabular}{|c|c|c|c|c|c|c|c|c|}
\hline $\begin{array}{c}\text { Doente } \\
n^{\circ}\end{array}$ & Idade & TARV & $\begin{array}{c}\text { Tempo de } \\
\text { diagnóstico de } \\
\text { infecção por VIH }\end{array}$ & $\begin{array}{l}\text { Doenças } \\
\text { oportunistas } \\
\text { prévias }\end{array}$ & $\begin{array}{l}\text { TCD4+ } \\
\text { (células/ } \\
\text { uL) }\end{array}$ & $\begin{array}{l}\text { CV VIH } \\
\text { (cópias/ } \\
\text { mL) }\end{array}$ & Estadiamento & $\begin{array}{l}\text { Evolução } \\
\text { (tempo de } \\
\text { sobrevida) }\end{array}$ \\
\hline 1 & 38 & Sim & 8 & - & 240 & n.d. & $0:$ Tis & Cura (4a) \\
\hline 2 & 60 & Não & 19 & - & 6 & $>500000$ & Não disponível & Óbito (12m) \\
\hline 3 & 40 & Sim & 17 & $\begin{array}{l}\text { Sarcoma de Kaposi } \\
\text { cutâneo }\end{array}$ & 930 & n.d. & II: T3NxMx & Cura (2a) \\
\hline 5 & 49 & Sim & 16 & - & 597 & n.d. & IIIA: T4NxMx & Óbito (12m) \\
\hline 6 & 32 & Sim & 18 & Tuberculose pulmonar & 200 & n.d. & II: T2NxMx & Cura (6a) \\
\hline 7 & 53 & $\operatorname{Sim}$ & 16 & - & 155 & n.d. & IIIA: T4NxMx & Óbito $(2 m)$ \\
\hline 8 & 81 & Sim & 16 & - & 566 & n.d. & IIIB: T4N3M0 & Cura (5a) \\
\hline
\end{tabular}

CV: carga viral; d: dias; m: meses; n.d.: não detectável; TARV: terapêutica antirretrovírica 
e falsas vontades $(n=2)$. Um doente apresentava história de abcesso anal recidivante, e um doente tinha história prévia de carcinoma espinocelular in situ. Um doente encontrava-se assintomático. Seis doentes tiveram condilomas perianais síncronos com/ou previamente ao diagnóstico de neoplasia do canal anal.

Todos os diagnósticos foram realizados por anuscopia com biópsia. O diagnóstico histólogico foi de carcinoma pavimentocelular em 9 doentes, um deles variante basalóide, a par de um caso de lesão intraepitelial pavimentosa de alto grau (ASIN-3). Foi realizada genotipagem de HPV a partir de material da peça de biópsia por amplificação por PCR e detecção por microassays em 8 casos, (Tabela 2), verificando-se presença de HPV em 5 casos, com estirpes de alto risco em 4 doentes. Em um doente foram detectados HPV 6 e 45 simultaneamente.

Tabela 2 - Genotipagem de HPV $(n=8)$.

\begin{tabular}{l|c}
\multicolumn{1}{c|}{ GENOTIPO HPV } & $\mathbf{n}$ \\
\hline HPV 16 & 3 \\
\hline Inconclusivo & 2 \\
\hline HPV 6, HPV 45 & 1 \\
\hline HPV 11 & 1 \\
\hline Negativo & 1 \\
\hline
\end{tabular}

O estadiamento de acordo com o sistema TNM revelou frequentemente doença avançada (Tabela 1). Não foi possível obter informação relativa a estadiamento do doente $n^{\circ} 3$, realizado noutra instituição de saúde.

$O$ tratamento envolveu cirurgia $(n=4)$, radioterapia $(n=4)$ e quimioterapia $(n=2)$. Registaram-se 4 óbitos relacionados com a neoplasia, encontrando-se em seguimento os restantes 6 doentes, com tempo livre de doença em média de 1,4 anos (0-6 anos). A sobrevida aos 5 anos foi de $40 \%$.

\section{DISCUSSÃO}

A incidência anual global de cancro anal é aproximadamente de 1-2/100.000 habitantes, estando substancialmente aumentada nos doentes $\mathrm{HSH}$ com infecção por $\mathrm{VIH}$. Nos EUA, a incidência de cancro anal nesta população é de 131/100.000, de 46/100.000 nos outros homens VIH e de 30/100.000 nas mulheres seropositivas para o $\mathrm{VIH}$.

Algumas séries recentes revelam que o número em excesso de casos de cancro do canal anal ultrapassa já o excesso de casos de cancro do pulmão em doentes $\mathrm{VIH}^{2}{ }^{2}$ uma das neoplasias não definidoras de SIDA mais frequentemente reportada. ${ }^{3-6}$ No nosso centro verificamos igualmente a presença desta tendência de evolução dos tipos de neoplasias nos doentes com infecção por $\mathrm{VIH}$, verificando-se actualmente um número de casos de cancro do canal anal muito próximo dos casos de neoplasia do pulmão (10 e 11 casos, respectivamente). $O$ envelhecimento e aumento da sobrevida dos doentes com infecção por VIH poderão estar relacionados com o aumento de neoplasias de SIDA que se tem verificado. ${ }^{4}$ Admite-se que também as recentes mudanças na prática clínica, com mais realização de exames de rastreio em doentes com infecção por $\mathrm{VIH}$, possam contribuir para esta tendência.

O cancro anal é primariamente causado pela infecção por estirpes de alto risco do vírus do papiloma humano. VIH e HPV influenciam-se reciprocamente não só pelo papel promotor que a infecção HPV tem na aquisição da infecção VIH, mas sobretudo pela maior prevalência e incidência de infecção por HPV em indivíduos com infecção $\mathrm{VIH}$, especialmente $\mathrm{HSH}$, que têm mais infecções e mais frequentemente por múltiplos serótipos, incluindo os de alto risco oncogénico. ${ }^{7-9}$ Assim, estima-se que mais de $80 \%$ dos $\mathrm{HSH}$ infectados pelo VIH têm infecção por $\mathrm{HPV}^{4}$ com uma prevalência de 22-34\% de infecção por HPV de alto risco. ${ }^{10}$ Estes achados relacionam-se com uma probabilidade cerca de 44 vezes maior de cancro do canal anal nesta população. ${ }^{10}$

Na nossa amostra, todos os indivíduos eram do sexo masculino, tendo-se conseguido confirmar epidemiologia sexual de risco na maioria dos casos, nomeadamente homens com múltiplos parceiros do mesmo sexo. Além desta, e da infecção por VIH, não foram apurados outros factores de risco.

Os sintomas clínicos mais frequentemente descritos são a rectorragia, a proctalgia, a presença de uma massa, história de alteração dos hábitos intestinais e tenesmo. ${ }^{10} \mathrm{Na}$ nossa amostra, a presença de condilomatose, rectorragia, dor anal/ perianal foram frequentes, sendo de salientar um diagnóstico de cancro anal estabelecido após citologia do canal anal com detecção de HPV 16 em doente assintomático.

Naqueles em que foi possível a genotipagem de HPV, metade apresentaram estirpes de alto risco, nomeadamente HPV 16 e 45. Nesta série, a sobrevida aos 5 anos é de $40 \%$, o que está de acordo com a sobrevida de $39-65 \%$ reportada na bibliografia.

Os dados são contraditórios no que diz respeito ao impacto da TARV na prevenção e evolução da infecção por HPV e neoplasias, não sendo claro o benefício da sua utilização precoce e/ou controlo virológico e imunológico. É possível que a reconstituição imunitária associada à TARV não influencie a história natural de infecções com HPV e cancro anal, uma vez que o aumento da sobrevida decorrente da utilização de TARV pode estar associada a um aumento da incidência de neoplasias que não se relacionam fortemente com a imunodepressão. ${ }^{6}$

Os estudos mais recentes em mulheres com infecção por VIH mostraram um efeito protector da TARV na presença de HPV no cérvix ${ }^{11}$ e progressão para $\mathrm{CIN}$, particularmente nas mulheres com supressão virológica, ${ }^{11}$ apoiando as recomendações internacionais de início de TARV em doentes com neoplasias relacionadas com HPV, independentemente dos estádios virológico e imunológico. Contudo, a heterogeniedade dos dados é grande relativamente a $\mathrm{HSH}$ e neoplasia anal. Um nadir baixo de linfócitos TCD4+ parece estar 
associado a maior risco de cancro anal invasivo em homens com infecção por VIH..$^{3,6,13}$ Em alguns trabalhos a TARV não pareceu ter um impacto significativo na persistência da infecção por HPV ou história natural de lesões pré-malignas, ${ }^{6}$ contudo outros autores verificaram um efeito significativo da utilização da TARV, principalmente quando era tido em conta o nadir de linfócitos TCD4.$+{ }^{14}$,Assim é possível que o risco aumentado de neoplasias relacionadas com infecção por HPV se possa dever a um estado prolongado de imunodepressão relativamente estável e não a imunossupressão de agravamento progressivo. ${ }^{6}$ Dado o intervalo de tempo que pode ocorrer entre a determinação do nadir de linfócitos TCD4+ correspondente ao diagnóstico de SIDA e o diagnóstico do cancro anal, não é claro qual o período do processo de oncogénese das neoplasias relacionadas com HPV em que a imunossupressão seja etiologicamente relevante, sendo possível que o impacto mais significativo seja por pobre controlo imunológico de lesões pré-malignas e não propriamente durante a progressão de doença invasiva. ${ }^{6}$ Desse modo, poder-se-ia justificar o aumento de incidência observado à medida que foi também aumentando a sobrevida destes doentes, fruto de uma cada vez maior disponibilidade da TARV. ${ }^{15}$ De facto, seria expectável, caso a TARV tivesse um papel realmente protector, uma vez que a sua utilização e disponibilidade têm vindo a aumentar globalmente, que a tendência observada fosse no sentido de observar uma diminuição da incidência de cancro anal, e não o contrário. Por outro lado, enquanto que em doentes com infecção por $\mathrm{VIH}$ a incidência de cancro anal tem vindo a aumentar, o mesmo não se verifica relativamente às incidências de cancro cervical, da orofaringe, pénis, vagina ou vulva. ${ }^{6}$

No conjunto de casos aqui apresentado, os óbitos verificaram-se em doentes com carga vírica detectável e indetectável ( $n=2$, respectivamente), três sob TARV, dois dos quais com CD4+ inferior a 250 células/uL (Tabela 1). O reduzido tamanho da nossa amostra e a ocorrência de óbitos em doentes com características tão díspares não permite retirar conclusões.

O nosso estudo possui algumas limitações. $\bigcirc$ reduzido número de casos e a impossibilidade de determinar com rigor a o tipo de epidemiologia sexual do universo global de doentes $\mathrm{HSH}$ em seguimento não permite a comparação entre o grupo de HSH com cancro do canal anal e os restantes $\mathrm{HSH}$ com infecção por VIH sem doença neoplásica. Não foi possível apurar pormenorizadamente a presença de factores de risco adicionais, como por exemplo o tabagismo, classicamente descrito como um factor de risco para neoplasias relacionadas com infecção por HPV. As determinações da contagem de linfócitos TCD4 + na altura do diagnóstico da infecção por VIH e no diagnóstico do cancro anal poderão não reflectir de modo adequado as flutuações no estado de imunossupressão, uma vez que não foi feita uma análise sistemática da evolução da contagem de linfócitos TCD4+ entre o diagnóstico de infecção por $\mathrm{VIH}$ e o diagnóstico de cancro do canal anal. Não foi feita uma análise detalhada da abordagem terapêutica, por se considerar fora do âmbito deste trabalho. Finalmente, o tamanho da nossa amostra e a incapacidade de quantificar precisamente o número de $\mathrm{HSH}$ com infecção por VIH em seguimento não nos permite calcular a incidência.

Algumas instituições recomendam o rastreio de lesões anais pré-malignas nestes doentes, de forma a prevenir o cancro invasivo, com recurso a técnicas de citologia do canal anal com ou sem métodos de detecção e tipificação de HPV, complementadas ou não por anuscopia de alta resolução. Infelizmente, estes métodos têm limitações importantes: dependendo do método, e se utilizada isoladamente como método de triagem de doentes com AIN, a tipificação anal do HPV tem uma boa sensibilidade mas baixa especificidade e baixo valor preditivo positivo, particularmente em $\mathrm{HSH}$ e $\mathrm{VIH}^{16}$; a citologia anal tem especificidades e sensibilidades muito variáveis (69-93\% para a sensibilidade e $32-59 \%$ para a especificidade) ${ }^{11}$ e a disponibilidade da anoscopia de alta resolução é limitada. ${ }^{17}$ A principal limitação da implementação de tais programas será o acesso a anoscopias, uma vez que é previsível que mais de metade dos $\mathrm{HSH}$ e $20-30 \%$ de mulheres com infecção $\mathrm{VIH}$ apresentem resultados anormais na citologia do canal anal, requerendo referenciação para realização de anoscopia de alta resolução. ${ }^{17}$ Outra limitação importante desta estratégia é a necessidade de multidisciplinaridade a nível hospitalar, obrigando a que fossem cumpridos prazos razoáveis na realização de exames, entrega de resultados, decisão e intervenção terapêuticas. A implementação de programas de rastreio teria sempre que ser acompanhada de informação e divulgação sobre a infecção por HPV nas populações alvo, sendo essencial a compreensão por parte dos doentes sobre a metodologia, áreas de incerteza e eventual necessidade de realização de procedimentos invasivos. Uma revisão recente da literatura ${ }^{16}$ sugere que a população alvo de programas de rastreio deverão ser indivíduos em elevado risco para o desenvolvimento de neoplasia do canal anal, particularmente HSH com infecção por VIH. De acordo com esta evidência, concordamos que o rastreio do cancro anal apenas fará sentido se estiver garantido o adequado seguimento de doentes com alterações na citologia do canal anal e que tais programas só devem ser implementados uma vez garantidos a participação de um laboratório de Anatomia Patológica com experiência na avaliação de citologias e biópsias do canal anal, assim como acesso fácil a anoscopias e tratamento nos casos em que este esteja indicado. $\bigcirc$ tratamento das lesões anais pré-cancerosas é muito invasivo, não existindo ainda consenso sobre como e quando tratar estas lesões, nem estudos randomizados que demonstrem que o tratamento diminui a incidência de cancro invasivo, aumente a sobrevida ou o tempo livre de doença. Por outro lado, existem análises de custo-efectividade que suportam o rastreio por rotina em determinados indivíduos em elevado risco para o desenvolvimento de cancro anal, particularmente $\mathrm{HSH}$ com infecção por $\mathrm{VIH} .{ }^{18}$

As vacinas contra o HPV têm eficácia demonstrada na prevenção da aquisição da infecção pelos tipos de HPV incluídos, redução da infecção genital e anal persistentes e 
doença neoplásica em mulheres e homens e, eventualmente, protecção na prevenção do cancro anal equiparável à observada na prevenção da infecção cervical por HPV. ${ }^{21-24}$ Apesar da informação sobre a eficácia destas vacinas na população VIH ser ainda escassa, existem já várias recomendações internacionais de vacinação contra o HPV em doentes com infecção por VIH que vêm enfatizar o potencial da vacinação na prevenção do cancro associado à infecção por HPV em doentes seropositivos. ${ }^{25-27}$ De acordo com as últimas recomendações americanas sobre prevenção e tratamento de doenças oportunistas em doentes com infecção por VIH, a vacinação contra o HPV está recomendada em todos os doentes com infecção por $\mathrm{VIH}$ com idades entre os 13-26 anos, na ausência de vacinação prévia na infância. A recente aprovação da vacina nonavalente contra o HPV (que inclui os tipos 31, 33, 45, 52 e 58, além dos já presentes na vacina quadrivalente) poderá vir a aumentar a cobertura vacinal.

\section{CONCLUSÕES}

Concluindo, na nossa população de doentes seropositivos para o VIH observámos um número importante de doentes com cancro anal. Estes eram todos homens, de idade moderadamente avançada, na sua quase totalidade $\mathrm{HSH}$, com infecção por $\mathrm{VIH}$ de longa data com controlo imunológico e TARV variáveis, conseguindo-se identificar infecção por HPV na biópsia de 5 doentes num total de 8 biópsias realizadas. O estádio III foi o mais frequentemente detectado, com necessidade de tratamento cirúrgico e radioterapia, e a sobrevida aos cinco anos foi de $40 \%$. Os nossos dados, à semelhança do que tem vindo a ser publicado, apontam para a necessidade de programas de rastreio consensuais e eficazes do cancro anal e, sobretudo, para uma necessidade de reforço da vacinação contra o HPV nos homens, nomeadamente nos $\mathrm{HSH}$, com ou sem infecção por VIH.

Conflitos de interesse: Os autores declaram não possuir conflitos de interesse. Suporte financeiro: $O$ presente trabaIho não foi suportado por nenhum subsídio ou bolsa. Confidencialidade dos dados: Os autores declaram ter seguido os protocolos do seu centro de trabalho acerca da publicação dos dados de doentes. Protecção de pessoas e animais: Os autores declaram que os procedimentos seguidos estavam de acordo com os regulamentos estabelecidos pelos responsáveis da Comissão de Investgação Clínica e Ética e de acordo com a Declaração de Helsínquia da Associação Médica Mundial.

Conflicts of interest: The authors have no conflicts of interest to declare. Financing Support: This work has not received any contribution, grant or scholarship. Confidentiality of data: The authors declare that they have followed the protocols of their work center on the publication of patient data. Protection of human and animal subjects: The authors declare that the procedures followed were in accordance with the regulations of the relevant clinical research ethics committee and with those of the Code of Ethics of the World Medical Association (Declaration of Helsinki).

\section{REFERÊNCIAS}

1. Riedel DJ, Tang LS, Rositch AF. The Role of Viral Co-Infection in HIV-Associated Non-AIDS-Related Cancers. Curr HIV/AIDS Rep. 2015 p12:362-72.

2. Robbins HA, Pfeiffer RM, Shiels MS, Li J, Hall HI, Engels EA. Excess cancers among HIV-infected people in the United States. J Natl Cancer Inst. 2015 6; 107. pii: dju503.

3. Worm SW, Bower M, Reiss P, Bonnet F, Law M, Fätkenheuer $G$, et al. Non-AIDS defining cancers in the D:A:D Study-time trends and predictors of survival: a cohort study. BMC Infect Dis. 2013; 13:471.

4. Chin-Hong PV, Palefsky JM. Natural history and clinical management of anal human papillomavirus disease in men and women infected with human immunodeficiency virus. Clin Infect Dis. 2002; 35:1127-34.

5. Patel P, Hanson DL, Sullivan PS, Novak RM, Moorman AC, Tong TC, et al. Adult and Adolescent Spectrum of Disease Project and HIV Outpatient Study Investigators. Incidence of types of cancer among HIV-infected persons compared with the general population in the United States, 1992-2003. Ann Intern Med. 2008; 148:728-36.

6. Chaturvedi AK, Madeleine MM, Biggar RJ, Engels EA. Risk of human papillomavirus-associated cancers among persons with AIDS. J Natl Cancer Inst. 2009; 101:1120-30.

7. Machalek DA, Poynten M, Jin F, Fairley CK, Farnsworth A, Garland SM, et al. Anal human papillomavirus infection and associated neoplastic lesions in men who have sex with men: a systematic review and meta-analysis. Lancet Oncol. 2012; 13:487-500.

8. Lissouba P, Van de Perre P, Auvert B. Association of genital human papillomavirus infection with HIV acquisition: a systematic review and meta-analysis. Sex Transm Infect. 2013; 89:350-6.

9. Houlihan CF, Larke NL, Watson-Jones D, Smith-McCune KK, Shiboski S, Gravitt PE, et al. Human papillomavirus infection and increased risk of HIV acquisition. A systematic review and meta-analysis. AIDS. 2012; 26:221122.

10. Brown B, Davtyan M, Galea J, Chow E, Leon S, Klausner JD. The role of human papillomavirus in human immunodeficiency virus acquisition in men who have sex with men: a review of the literature. Viruses. 2012; 4:3851-8.

11. Fife KH, Wu JW, Squires KE, Watts DH, Andersen JW, Brown DR. Prevalence and persistence of cervical human papillomavirus infection in HIV-positive women initiating highly active antiretroviral therapy. J Acquir Immune Defic Syndr. 2009; 51:274-82

12. Minkoff H, Ahdieh L, Massad LS, Anastos K, Watts DH, Melnick $S$, et al. The effect of highly active antiretroviral therapy on cervical cytologic changes associated with oncogenic HPV among HIV-infected women. AIDS. 2001; 15:2157-64.

13. Chiao EY, Hartman CM, El-Serag HB, Giordano TP. The impact of HIV viral control on the incidence of 
HIV-associated anal cancer. J Acquir Immune Defic Syndr. 2013; 63:631-8.

14. Conley L, Bush T, Darragh TM, Palefsky JM, Unger ER, Patel $P$, et al. Study to Understand the Natural History of HIV and AIDS in the Era of Effective Therapy (SUN Study) Investigators. Factors associated with prevalent abnormal anal cytology in a large cohort of HIV-infected adults in the United States. J Infect Dis. 2010; 202:1567-76.

15. vanVan der Snoek EM, van der Ende ME, den Hollander JC, Schutten M, Neumann HA, van Doornum GJ. Use of highly active antiretroviral therapy is associated with lower prevalence of anal intraepithelial neoplastic lesions and lower prevalence of human papillomavirus in HIV-infected men who have sex with men. Sex Transm Dis. 2012; 39:495-500.

16. de Pokomandy A, Rouleau D, Ghattas G, Trottier H, Vézina $S$, Coté $P$, et al. HAART and progression to high-grade anal intraepithelial neoplasia in men who have sex with men and are infected with HIV. Clin Infect Dis. 2011; 52:1174-81.

17. Piketty C, Selinger-Leneman H, Grabar S, Duvivier C, Bonmarchand $M$, Abramowitz $L$, et al. Marked increase in the incidence of invasive anal cancer among HIV-infected patients despite treatment with combination antiretroviral therapy. AIDS. 2008; 22:1203-11.

18. Smyczek $P$, Singh AE, Romanowski B. Anal intraepithelial neoplasia: review and recommendations for screening and management. Int J STD AIDS. 2013; 24:843-51.

19. Wilkin TJ. Screening for anal cancer: Who, when, and how. Medscape. Apr 22, 2010. [consultado em 16 Fevereiro 2014] Disponível em http://www.medscape.com/ viewarticle/720388

20. Goldie SJ, Kuntz KM, Weinstein MC, Freedberg KA, Welton ML, Palefsky JM. The Clinical Effectiveness and Cost-effectiveness of Screening for Anal Squamous Intraepithelial Lesions in Homosexual and Bisexual HIV-Positive Men. JAMA. 1999; 281:1822-9.

21. Kreimer AR, González P, Katki HA, Porras C, Schiffman $M$, Rodriguez AC, et al; CVT Vaccine Group. Efficacy of a bivalent HPV 16/18 vaccine against anal HPV 16/18 infection among young women: a nested analysis within the Costa Rica Vaccine Trial. Lancet Oncol. 2011 ; 12:862-70.

22. Garland SM, Hernandez-Avila M, Wheeler CM, Perez G, Harper DM, Leodolter S, et al; Females United to Unilaterally Reduce Endo/Ectocervical Disease (FUTURE) I Investigators. Quadrivalent vaccine against human papiIlomavirus to prevent anogenital diseases. N Engl J Med. 2007;356:1928-43.

23. Palefsky JM, Giuliano AR, Goldstone S, Moreira ED Jr, Aranda $C$, Jessen $H$, et al. HPV vaccine against anal HPV infection and anal intraepithelial neoplasia. $N$ Engl J Med. 2011; 365:1576-85. doi: 10.1056/NEJMoa 1010971.

24. Giuliano AR, Palefsky JM, Goldstone S, Moreira ED Jr, Penny ME, Aranda C, Vardas E, Moi H, Jessen H, Hillman R, Chang YH, Ferris D, Rouleau D, Bryan J, Marshall JB, Vuocolo S, Barr E, Radley D, Haupt RM, Guris D. Efficacy of quadrivalent HPV vaccine against HPV Infection and disease in males. N Engl J Med. 2011 Feb 3; 364(5):401-11. doi: 10.1056/NEJMoa0909537.

25. National Center for Immunization and Respiratory Diseases. General recommendations on immunization - recommendations of the Advisory Committee on Immunization Practices (ACIP). MMWR Recomm Rep. 2011 Jan 28; 60(2):1-64. Disponível a 16 de Fevereiro de 2014 em http://www.cdc.gov/mmwr/preview/mmwrhtml/ rr6002al.htm

26. European Aids Clinical Society. European Guidelines for treatment of HIV-infected adults in Europe. Disponível a 6 de Outubro de 2015 em http://www. eacsociety.org/files/2015_eacsguidelines_8.0-english_ revised-20151104.pdf.

27. Medicine Association of the Infectious Diseases Society of America. Panel on Opportunistic Infections in HIV-Infected Adults and Adolescents. Guidelines for the prevention and treatment of opportunistic infections in HIV-infected adults and adolescents: recommendations from the Centers for Disease Control and Prevention, the National Institutes of Health, and the HIV [consultado 10 Maio de 2015] Disponível emhttp://aidsinfo.nih.gov/contentfiles/ Ivguidelines/adult_oi.pdf . 